\title{
Online dimensional control of rolled steel profiles using projected fringes
}

\author{
Kjell J. Gåsvik ${ }^{1}$ (D) Kjell G. Robbersmyr ${ }^{1} \cdot$ Trond Vadseth $^{2}$ \\ Received: 23 August 2019 / Accepted: 5 February 2020 /Published online: 7 March 2020 \\ (C) The Author(s) 2020
}

\begin{abstract}
Fringe projection is a versatile method for mapping the topography of surfaces. In this paper, it is used to measure the defects on the head of railroad rails while the rails are moving. Railroad rails are made by hot rolling. The quality of the finished product is generally good, but surface texture will deteriorate with increasing temperature. A method for online inspection therefore is very desirable. In the present experiment, dimensional inspection of the railroad rails was made online while moving at a speed of 1$2 \mathrm{~m} / \mathrm{s}$. Therefore, it is important to minimize the registration time. To achieve this, we apply a method of fringe location with subpixel accuracy that requires only a single exposure for each registration.
\end{abstract}

Keywords Fringe projection · Optical measurements $\cdot$ Railroad rails

\section{Introduction}

Projected fringes are a full-field method for mapping the topography of surfaces [1-13]. It consists of imaging the fringes projected onto the surface with subsequent processing of the fringe data. In recent years, it has been combined with phase shift techniques [14-19] or Fourier transform methods [20-27].

In this paper we describe an intensity method based on location of the fringe positions with sub-pixel accuracy by means of a zero-crossing algorithm. This method is applied to online measurement of dimensional errors of railroad rails. In the present experiment, the rails moved with a speed of 1-2 $\mathrm{m} / \mathrm{s}$. The processing time must therefore be reduced to a minimum.

It seems appropriate to compare our method with phase shift methods which also use projected fringes. Instead of measuring the intensity, these methods are based on measuring the phase.

Kjell J. Gåsvik

kgasvik@goose.no

1 Department of Engineering Science/Mechatronics, Faculty of Engineering and Science, University of Agder,

N-4886 Grimstad, Norway

2 SINTEF, division of Safety and Reliability, N-7465 Trondheim, Norway
(1) Phase shift methods require at least 3 (5 is most common) separate exposures of each deformation state. In addition, an additional operation for unwrapping the phase maps $[28,29]$ is required. Therefore, these methods become inferior when recording fast dynamic events. One benefit of our system is that only one single exposure is required for each deformation state.

(2) Divergent illumination introduces a non-linear phase into the fringe function, a fact also pointed out by refs $[1,7,9$, 17]. This makes phase methods more prone to errors.

(3) Our method is insensitive to image saturation. Saturation results in signal clipping, making the fringes non-sinusoidal. Phase shift methods rely on sinusoidal fringes, and the influence of such clipping is measured in [30].

(4) Our method is not dependent on correcting the lenses for image distortion. This is because both the reference and object surface are imaged by the same "distorted" lens.

\section{System description}

\subsection{Theory}

The measuring principle is illustrated in Fig. 1. Here, fringes with an inter-fringe distance $d$ are projected onto the xy-plane (the reference surface) under an angle $\theta_{1}$ to the $z$-axis and viewed (by e.g. a camera focused at infinity) under an angle 


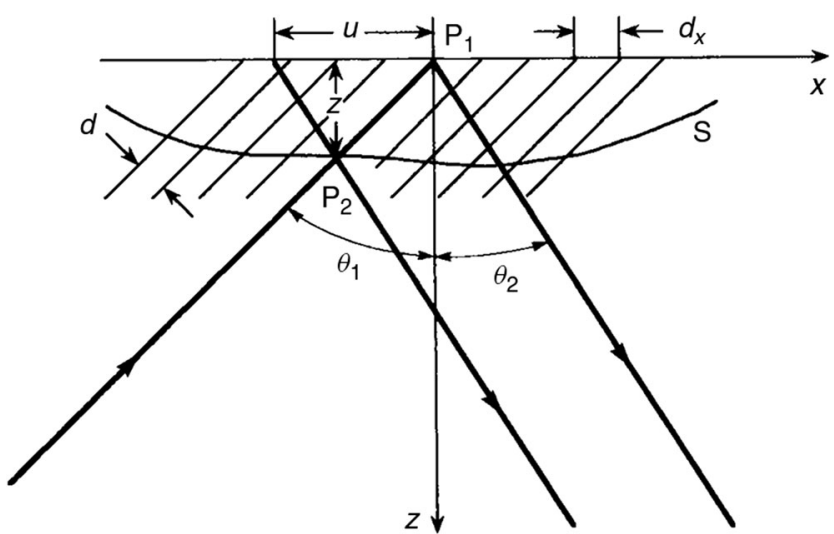

Fig. 1 The measuring principle. $\theta_{1}=$ projection angle. $\theta_{2}=$ viewing angle

$\theta_{2}$. Also, in the figure is drawn a curve $\mathrm{S}$ representing a surface to be measured. From the figure, we see that a fringe positioned at $P_{1}$ on the xy-plane will be displaced to $P_{2}$ on $\mathrm{S}$. This displacement is given by

$u=z\left(\tan \theta_{1}+\tan \theta_{2}\right)$

where $z$ is the height of $P_{2}$ above the xy-plane.

When imaging a grating of period $d_{g}$, the fringe period on the reference surface is given by

$d_{x 0}=m_{p} \frac{d_{g}}{\cos \theta_{1}}$

where $m_{p}$ is the magnification of the projection unit. When putting $u=d_{x 0}$, we find the surface deviation per fringe.

$z($ per fringe $)=\frac{m_{p} d_{g}}{\sin \theta_{1}}$

Figure 2 shows a more general situation where the projector emits divergent light, and the camera image the surface at a finite distance along the $z$-axis. When looking at an arbitrary

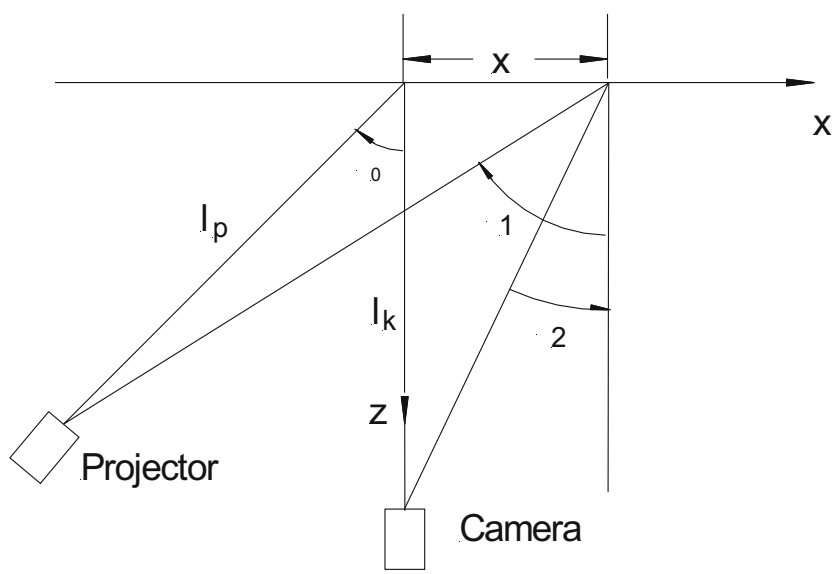

Fig. 2 Fringe projection geometry point $x$ on the xy-plane, we see from the figure that

$\tan \theta_{1}=\frac{l_{p} \sin \theta_{0}+x}{l_{p} \cos \theta_{0}}$

$\tan \theta_{2}=\frac{-x}{l_{k}}$

By inserting Eq. (4a) and (4b) into Eq. (1), we get

$$
\begin{aligned}
u & =z\left[\frac{l_{p} \sin \theta_{0}+x}{l_{p} \cos \theta_{0}}-\frac{x}{l_{k}}\right] \\
& =\frac{z}{\cos \theta_{0}}\left[\sin \theta_{0}+\frac{\left(l_{k}-l_{p} \cos \theta_{0}\right) x}{l_{p} l_{k}}\right]
\end{aligned}
$$

or

$z(x)=\left[\sin \theta_{0}+\frac{\left(l_{k}-l_{p} \cos \theta_{0}\right) x}{l_{p} l_{k}}\right]^{-1} u$

where $l_{k}$ is the distance between the camera lens and the coordinate center and $l_{p}$ is the distance between the projector and the coordinate center. To calibrate the system, one have to measure the quantities $l_{k}, l_{p}$, and $\theta_{0}$. To determine $\theta_{0}$, it is easiest to measure the distance between the lenses of the projector and the camera, and then calculate $\theta_{0}$ from the resulting triangle.

The relation given in Eq. (4) can be written as

$z(x)=S\left(\theta_{0}, l_{k}, l_{p}, x\right) \cdot u(x)$

where

$S\left(\theta_{0}, l_{k}, l_{p}, x\right)=\cos \theta_{0}\left[\sin \theta_{0}+\frac{\left(l_{k}-l_{p} \cos \theta_{0}\right) x}{l_{k} l_{p}}\right]^{-1}$

is a system function determined by the geometry of the experimental setup. Therefore, $S$ can be used as a look-up table. Notice when the camera and the projector are located at the same height above the reference surface $\left(l_{k}-l_{p} \cos \theta_{0}=0\right), S$ becomes equal to $1 / \tan \theta_{0}$, i.e. independent of $x$.

The measurement accuracy is dependent on the accuracy with which the fringe positions are detected. This is done by a zero-crossing algorithm described in [1, p 274]. By this algorithm, the fringe positions are located with sub-pixel accuracy [31].

\subsection{Measurement procedure}

It starts with calibrating the system as described above. Then the object and reference surfaces are recorded and stored as an array of $512 \times 512$ pixels, 8-bit deep, i.e. with intensity levels between 0 and 255 . Then, the fringe positions on the object and reference images are located, which determines $u$ and 
Fig. 3 Result from measurement of a rotation of a plane. The thick curve displays the measurement; the thin curve is the ideal straight line

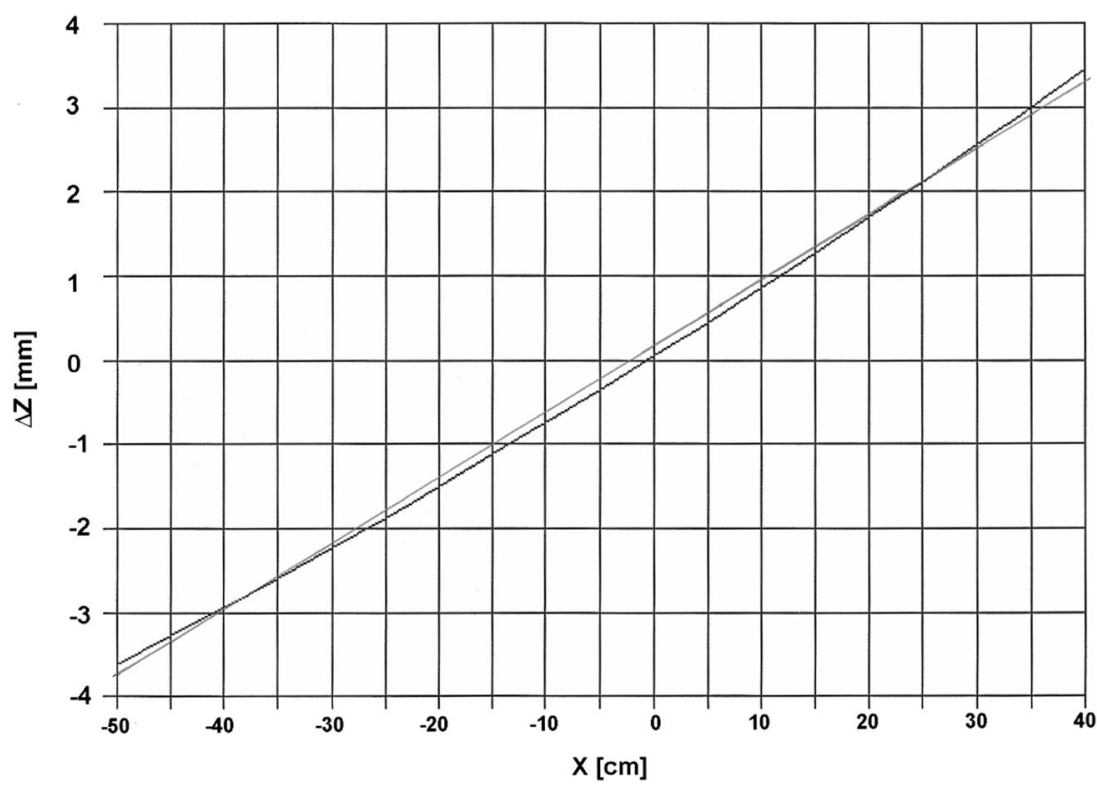

calculates $z$ from Eq. (6). The image of the reference surface is stored in memory once and for all. $u$ must be equal to the difference between mutual fringe orders on the reference and the object. In the general case, the zero-order fringe must be located manually. In the present project, this is not a problem. When passing the edge of a defect, $|u|$ will always be less than $d_{x} / 2$. In this situation, the object fringe is always closest to the corresponding reference fringe. The measurement algorithm therefore starts automatically without manual assistance. The calculated topography of the object surface can be stored in computer memory and be displayed as a grey scale-level picture or a contour map.

A simple and reliable test of this system is to image a plane surface before and after a small tilt. Figure 3 shows the result of such a test. A plane surface of about $1 \mathrm{~m}^{2}$ is imaged before and after a rotation angle of about 8 millirads about the vertical axis. From the smoothness of the measured curve, we can conclude that the zero-crossing algorithm works properly. Also, in the diagram is drawn the ideal straight line. The deviation from this line is seen to be less than about $0.1 \mathrm{~mm}$. This deviation is mainly due to small errors in the setup parameters resulting in a minor error in the system function. It should be noted that the projected fringes in this test had the highest possible quality.

\section{Experiments}

\subsection{Experimental setup}

Figure 4 shows the experimental setup. The railroad rail is mounted on a slide which can be moved in the $x$-direction.
The projector distance $l_{p}$, the grating period, and the projection angle were selected to give a grating pitch along the rail (the sampling distance) equal to $5 \mathrm{~mm}$. The acquisition time was $40 \mathrm{~ms}$ and the processing time was $50 \mathrm{~ms}$, giving a total sampling time $T=90 \mathrm{~ms}$. With a camera field of view equal to $w$, the speed of the rail must be $v<w / T$. In our experiment, we set $w=200 \mathrm{~mm}$, which means that the speed must be less than $2.2 \mathrm{~m} / \mathrm{s}$. When this condition is fulfilled, the dimensions of the rail will be continuously monitored at a sampling interval of $5 \mathrm{~mm}$ in the $x$-direction. By processing every 12 th horizontal line of the image, the sampling interval will be $5 \mathrm{~mm}$ also in the $y$-direction. This means that an array of $512 \times 42$ pixels are being processed. The result is that the dimensions of the rail will be checked at every point in an array of $40 \times 42$ points. To visualize the result, every point of the array where the dimension (surface height) deviate from the ideal shape by a magnitude exceeding a selectable threshold, the actual point will lit up on the monitor. In this experiment, this threshold was set to $0.4 \mathrm{~mm}$.

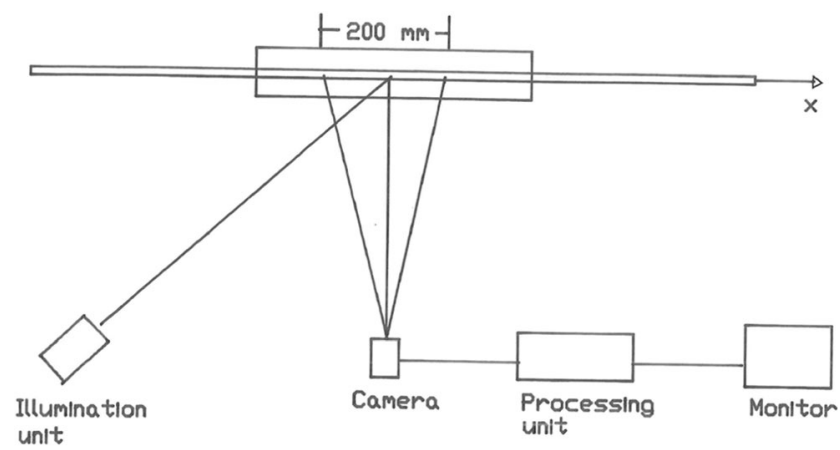

Fig. 4 The experimental setup 


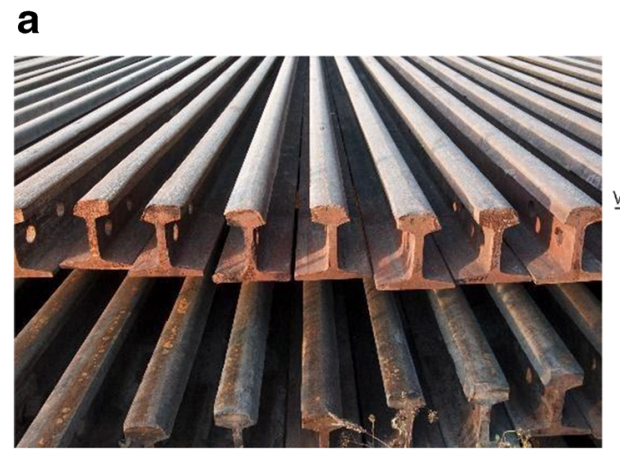

b

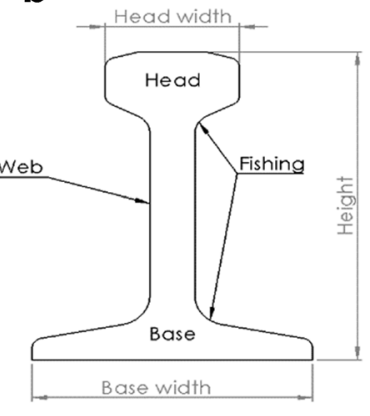

C

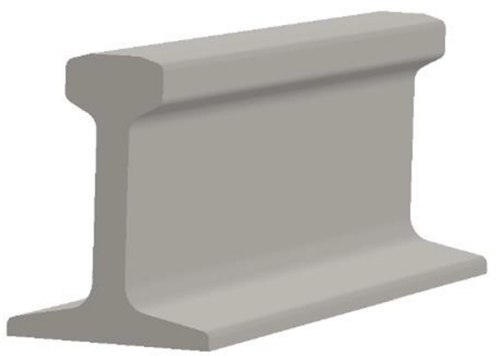

Fig. 5 a The product after the roll forming process. Illustration photo: colourbox.no. b Definition of the different parts. c Cross section of the railroad rail

Before the measurement sequence started, a $200 \mathrm{~mm}$ long defect-free part of the rail was imaged and stored in memory. This initial image served as the reference for the subsequent images in the online sequence. The contrast of the projected fringes was satisfactory without any preparation of the surface. The speed of the rails was 1$2 \mathrm{~m} / \mathrm{s}$.

\subsection{Specimen description}

Railway rails are produced by a hot rolling process. They can be up to $120 \mathrm{~m}$ long and should satisfy very strict tolerances required by international standards. These are related both to straightness and defects on the surface.

Figure 5 shows a cross section of railroad rail with the definition of the head, the web, and the base, and also a section of a railroad rail after the roll forming process is shown.

In this project, 3 samples of rolled railroad rails of different lengths were investigated:

- Sample A: length $=1000 \mathrm{~mm}$

- Sample B: length $=500 \mathrm{~mm}$

- Sample C: length $=460 \mathrm{~mm}$

The location of the defects on the rails is marked in Figs. 6 and 7 .

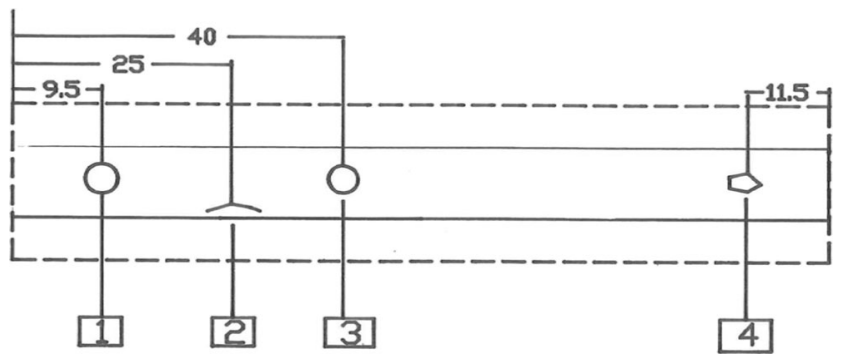

Fig. 6 Approximate location of the dimensional error on sample A. Errors no. 2 and no. 3 are shown in Fig. 8. Distances are in $\mathrm{cm}$

\subsection{Experimental results}

Some of our results are shown in Figs. 8 and 9. The rails moved with a speed of $1-2 \mathrm{~m} / \mathrm{s}$. The measurements are done on the top of the head of the rails.

From Fig. 9, it can be seen that both the position and the shape of the dimensional errors are clearly indicated on the monitor.

\section{Discussion}

Our results show that we have successfully detected the shape and positions of the dimensional errors on samples of railroad rails. Even small errors such as a small hole of approx. $1 \mathrm{~cm}$ (error no. 3 on sample A) and a small notch (error 2 on sample A) are detected. To have continuous inspection, our setup could measure errors with a speed of the rail up to $2.2 \mathrm{~m} / \mathrm{s}$. As mentioned in Sec. 3.1, the speed can be increased by increasing the camera field of view. As an example, with a field of view of $500 \mathrm{~mm}$, the speed could be increased up to $5.5 \mathrm{~m} / \mathrm{s}$.

It is clear that our experiment has not included the possibility that the rail can move sideways as a whole. We are presently building up a new system where we hopefully can solve this problem. We have however some ideas how to do it. As can be seen in Figs. 8 and 9, fringes are also projected on the base of the rail. These fringes can probably monitor the movement in the $z$-direction. Our goal in the present experiment was to test if our method was able to detect defects on the head of the rails while moving. This was not self-evident in advance.

\section{Conclusion}

A method for full-field dimensional control of moving rolled steel profiles is presented. The experimental results were successful in mapping the dimensional errors of the railroad rails online when it moves with a speed of approximately $2 \mathrm{~m} / \mathrm{s}$. 
Fig. 7 Approximate location of the dimensional error on a sample $\mathrm{B}$ and $\mathbf{b}$ sample C. Distances are in $\mathrm{cm}$

Fig. 8 Dimensional error on sample A. a Error no. 3. b Error no. 2. (The pictures of error nos. 1 and 4 are not included) a

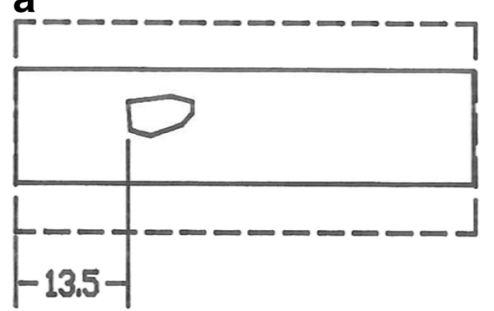

a

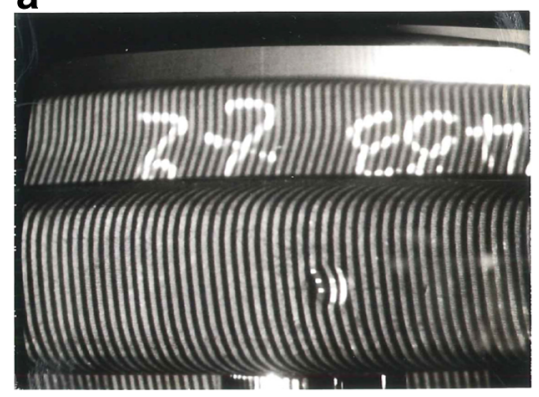

a

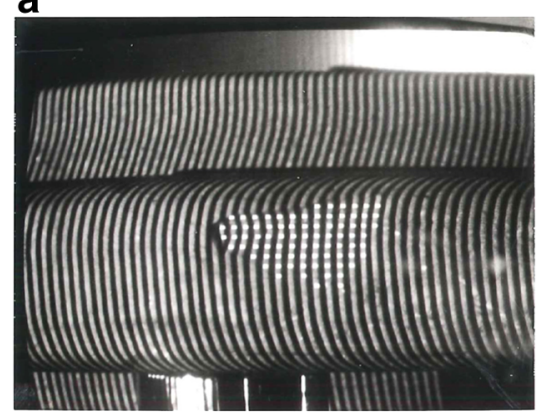

b

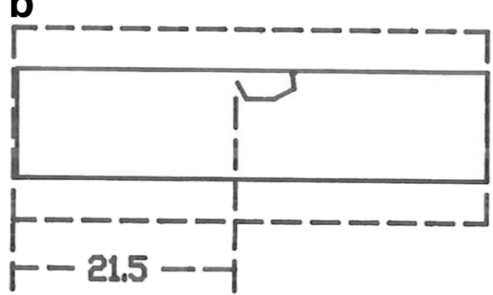

Fig. 9 Dimensional error on a sample B and b sample $\mathrm{C}$
Acknowledgements Open Access funding provided by University of Agder.

Open Access This article is licensed under a Creative Commons Attribution 4.0 International License, which permits use, sharing, adaptation, distribution and reproduction in any medium or format, as long as you give appropriate credit to the original author(s) and the source, provide a link to the Creative Commons licence, and indicate if changes were made. The images or other third party material in this article are included in the article's Creative Commons licence, unless indicated otherwise in a credit line to the material. If material is not included in the article's Creative Commons licence and your intended use is not permitted by statutory regulation or exceeds the permitted use, you will need to obtain permission directly from the copyright holder. To view a copy of this licence, visit http://creativecommons.org/licenses/by/4.0/.

\section{References}

1. Gåsvik KJ (2002) Optical metrology, 3rd edn. Wiley, Chichester ISBN 0-470-84300-4

2. Sciammarella C et al (2008) General model for moire contouring, part 1: theory. Opt Eng 47:033605-033620

3. Chen F, Brown GM, Song M (2000) Overview of threedimensional shape measurement using optical methods. Opt Eng 39:10-22

4. Gorthi SS, Rastogi P (2010) Fringe projection techniques: "whither we are". Opt Lasers Eng 48:133-140

5. Su X, Zhang Q (2010) Dynamic 3-D shape measurement method: a review. Opt Lasers Eng 48:191-204

6. Robbersmyr KG, Gåsvik KJ, Vadseth T, Karimi HR (2017) Surface topography measurement of double-curved propeller blades using projected fringes. Int J Manuf Technol 91:375-381 
7. Wang $\mathrm{Z}, \mathrm{Du} \mathrm{H}, \mathrm{Bi} \mathrm{H}$ (2006) Out-of-plane shape determination in generalized fringe projection profilometry. Opt Express 14:1212212133

8. Huntley JM, Ogundana T, Borguete RL, Coggrave CR (2007) Large-scale full-field metrology using projected fringes: some challenges and solutions. SPIE 06:6616

9. Martinez A et al (2010) Iterative estimation of the topography measurement by fringe-projection method with divergent illumination by considering the pitch variation along the z-direction. Opt Lasers Eng 48:877-881

10. Gåsvik KJ, Robbersmyr KG, Vadseth T (2010) Projected fringes for the measurement of large aluminum ingots. Meas Sci Technol 21: 105302

11. Gåsvik KJ et al (2014) Deformation measurement of circular steel plates using projected fringes. Int J Adv Manuf Technol 70(1):321326

12. Zhang S (2010) Recent progresses on real-time 3-D shape measurement using digital fringe projection techniques. Opt Lasers Eng 48: $149-158$

13. Zang S (2018) High-speed 3d shape measurement with structured light methods. A review. Opt Lasers Eng 106:119-131

14. Jeong M-S, Kim S-W (2001) Phase-shifting projection moire for out-of-plane displacement measurement. Proc SPIE 4317:170-179 Second International Conference on Experimental Mechanics

15. Yoshizawa T, Tomosawa T (1993) Shadow moiré topography by means of phase-shift method. Opt Eng 32(7):1668-1674

16. Huang P, Chiang FP (1999) Recent advances in fringe projection technique for 3D shape measurement. SPIE Conference on Optical Diagnostics for Fluids/Heat/Combustion and Photomechanics for Solids, Colorado, vol 3783

17. Chen L, Quan C (2005) Fringe projection profilometry with nonparallel illumination: a least-squares approach. Opt Lett 30(16): 2101-2103

18. Sansoni G, Trebeschi M, Docchio F (2006) Fast 3 D profilometer based upon the projection of a single fringe pattern and absolute calibration. Meas Sci Technol 17:1757-1766

19. Huang P et al (2005) Trapezoidal phase shifting for threedimensional shape measurement. Opt Eng 44(12):123601-123609
20. Takeda M, Ina H, Kobayashi S (1982) Fourier-transform method of fringe-pattern analysis for computer-based topography and interferometry. Opt Soc Am 72(1):156-160

21. Takeda M, Mutoh K (1983) Fourier transform profilometry for the automatic measurement of 3-D object shape. Appl Opt 24:39773982

22. Spagnolo $\mathrm{G}$ et al (2000) Contouring of artwork surface by fringe projection and FFT analysis. Opt Lasers Eng 33:141-156

23. Huang $L$ et al (2010) Comparison of Fourier transform, windowed Fourier transform, and wavelet transform methods for phase extraction from a single fringe pattern in fringe projection profilometry. Opt Lasers Eng 48:141-148

24. Sciammarella C, Lamberti L, Sciammarella FM (2005) Highaccuracy contouring using projection moire. Opt Eng 44(9): 093605-093617

25. Salas L, Luna E, Salinas J, Garcia V (2003) Profilometry by fringe projection. Opt Eng 42:3307-3314

26. Malcom AA, Burton DR, Lalor MJ (1990) Full-field Fourier fringe analysis for industrial inspection. SPIE, $(08 / 1990)$

27. Sciammarella $C$ et al (2008b) General model for moire contouring, part 2: applications. Opt Eng 47:033606-033622

28. Zappa E, Busca G (2008) Comparison of eight unwrapping algorithms applied to Fourier-transform profilometry. Opt Lasers Eng 46:106-116

29. Zuo C, Huang L, Zhang M, Chen Q, Asundi A (2016) Temporal phase unwrapping algorithms for fringe projection profilometry. A comparative review. Opt Lasers Eng 85:84-103

30. Waddington C, Kofman J (2014) Modified sinusoidal fringe-pattern projection for variable illuminance in phase-shifting three-dimensional surface shape metrology. Opt Eng 53:84109

31. Gåsvik KJ, Robbersmyr KG (1994) Error analysis of a zerocrossing algorithm for fringe location. Opt Eng 33:246-250

Publisher's note Springer Nature remains neutral with regard to jurisdictional claims in published maps and institutional affiliations. 